\title{
COMPLIANT PRESS-FIT TECHNOLOGY INTEGRATED INTO A NEW BACK PANEL PACKAGING APPROACH ${ }^{\dagger}$
}

\author{
A. KERKHOFF \\ Berg Electronics 's-Hertogenbosch Netherlands \\ (Received November 25, 1982; in final form May 25, 1983)
}

\begin{abstract}
Involving, as it does, DIN and Reversed DIN board connectors, compliant press-fit pins, round cable, flat cable, and coaxial-connectors, the Berg Backpanel System has, over the past two years, gained a recognized position in the European and US markets.

Development and expansion of the system in depth, and in breadth, is still ongoing. One of the major subjects is press-fit: preloaded press-fit connectors and studies focused on the relationship between printed circuit board properties vs. compliant press-fit pin design.

This paper presents results of these developments and discusses design options.
\end{abstract}

\section{INTRODUCTION}

In the electronics industry in Europe, the two-part board connector according to IEC 603-2 and DIN 41612 has proved its usefulness and reliability in a wide variety of board-to-board connection applications. Recently, Du Pont Berg Electronics has added new products and features designed to further increase packaging versatility.

A new addition to the DIN 41612 family is the reversed DIN connector (R series see Figure 1). Compared to the C-series, 3-row, 0.100" connectors, the R-series has a number of advantages. These are:

(a) Cost effectiveness is improved. Racks are often sub-equipped, i.e. some of the backpanel connectors are not used. In the case of Reversed DIN, the backpanel uses the less expensive, male connector half.

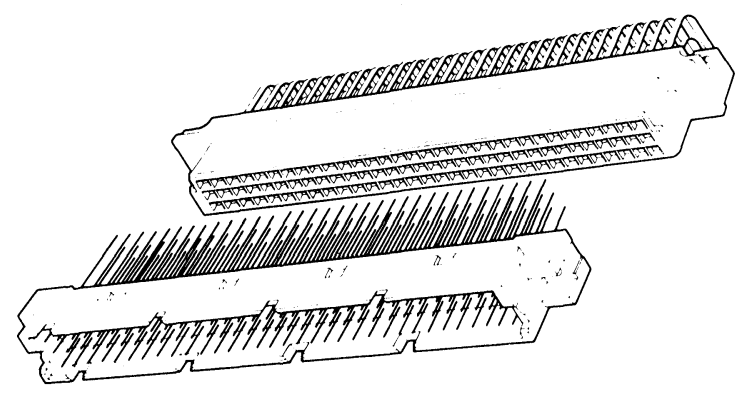

FIGURE 1 Reversed DIN connector. 1982.

$\dagger$ Paper originally presented at the Technical Sessions programme of Electronica, Munich, November, 

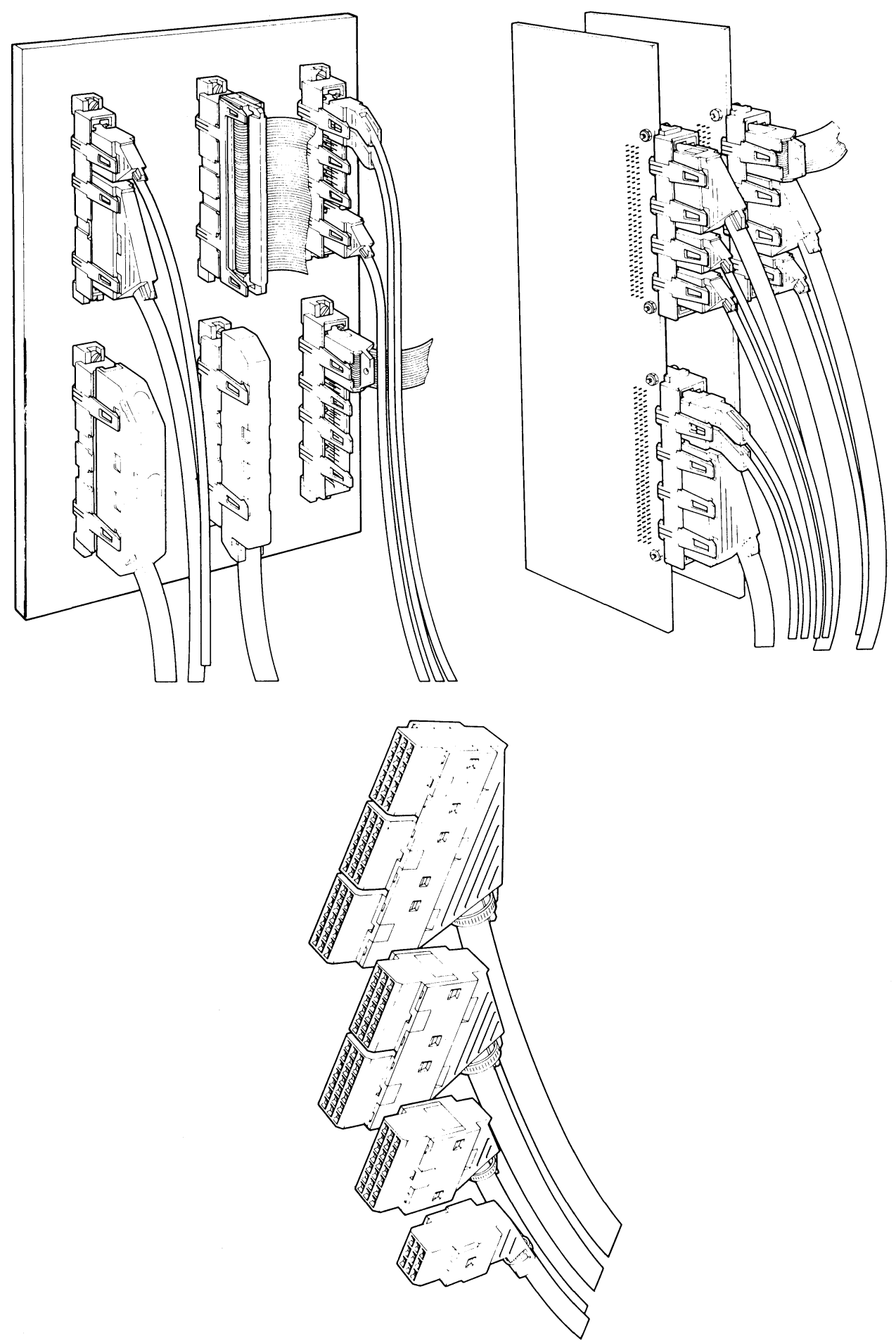

FIGURE 2 Examples of connectors. 
(b) The female connector, on the daughterboard, is subjected to more rough handling than the male part. However, the female receptacles are fully protected inside the plastic housing.

(c) The male half can easily be replaced by press-fit pins or a preloaded press fit male connector. Repairability is greatly improved when pins are used.

Cable connectors are also being used for input-output purposes and for connections between daughtercards or subracks. The Berg Backpanel System includes an extensive range of modular round and flat cable connectors and coax connections, all complying with the German PTT specification (494 G - see Figure 2). The 3-row, round cable connectors (0.100" pitch) use a cost and time saving Insulation Displacement Contact technique. A 64-position IDC connector is also available $(0.100 " \times 0.200$ " grid) with a very low profile, ideal where space is limited.

\section{PRESS FIT PINS}

The combined use of a reversed DIN female connector and press-fit pins has several design and cost advantages. These are:

(a) Improved reliability of damaged pins in a backpanel.

(b) Selective pin insertion - using only those pins that actually have a function can cut costs.

(c) Elimination of a costly soldering step in the board assembly process.

The first press-fit pins on the market were in fact solid with a rigid, oversize section, that was a force-fit into the p.c. board and consequently likely to put stress on it. This is obviously undesirable, especially since the number of press-fit pins in the panel can be as high as 8000 , and costly double sided and multilayer boards are being used more and more. Moreover, hole dimension tolerances for a solid pin are very narrow and difficult to achieve. The answer to these problems is a compliant press-fit pin which, when pressed into the board, copes with the forces through deformation. This has the following advantages:

(a) no damage to the p.c. board;

(b) lower insertion forces;

(c) a relatively constant retention force over a broad range of hole diameters;

(d) improved repairability.

\section{DESIGN CRITERIA FOR A PRESS FIT SYSTEM}

A trouble free press-fit system can only be obtained if the combination of p.c. board and pin is well chosen. Not only is the press-fit pin design critical, several parameters of the printed circuit board will have to be tightly controlled as well. Some specific design criteria and restrictions are as follows:

(a) Pin compliance should conform to specified hole tolerance.

(b) The degree of compliance determines the balance between insertion/retention forces and the degree of deformation of the hole plating or base material.

(c) Pin stability depends on the way its upper and lower halves are free to move independently. This determines the susceptibility of the pin to bowing when inserted. The ultimate true position of the pin tip also depends on the relative positioning of the different layers in multilayer p.c. boards. 
(d) The length of the compliant zone partly influences retention force and must also be a measure for the stability.

(e) Ideally, the terminal should be suitable for printed circuit boards of different thickness (e.g. 1,6 to $3,2 \mathrm{~mm}$ ). This calls for a press-fit section that has the same minimum performance over a range of insertion depths.

When the pins are used in a preloaded press-fit connector (Figure 3) several additional requirements should be considered:

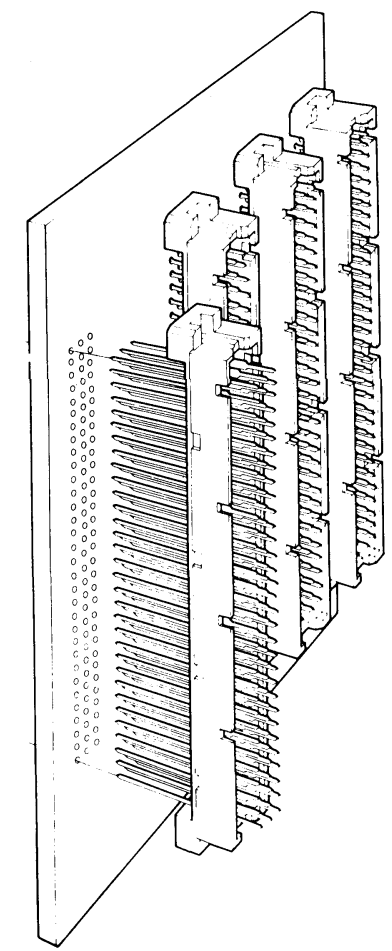

FIGURE 3 Preloaded press-fit connector.

(f) Serious tolerance problems can arise from mismatching of the p.c.b. hole pattern and the pin pattern in the connector body, with bent pins, high insertion forces and possible p.c.b. damage as a result.

(g) The preloaded connector - unlike individual pins - is inserted from the "machine side" of the backpanel, so the shoulder of the pin is on the opposite side. To allow repair of individual pins, special provisions must be made in the design of the press-fit connector's pins and body.

(h) The forces needed to press a 96 position connector into the board can easily exceed 1 ton. Obviously, the delicate p.c. board must be supported locally by the application machine; and a slightly diminished insertion force (per pin) is essential compared to that for a free standing press-fit pin.

In mastering this new technology, a two step approach has been used. First a "free standing" compliant pin has been designed. To ob tain the desired mating part for a female board connector or cable connector, the pins are stacked into the printed circuit board and a plastic housing is placed over them (Figure 4). 


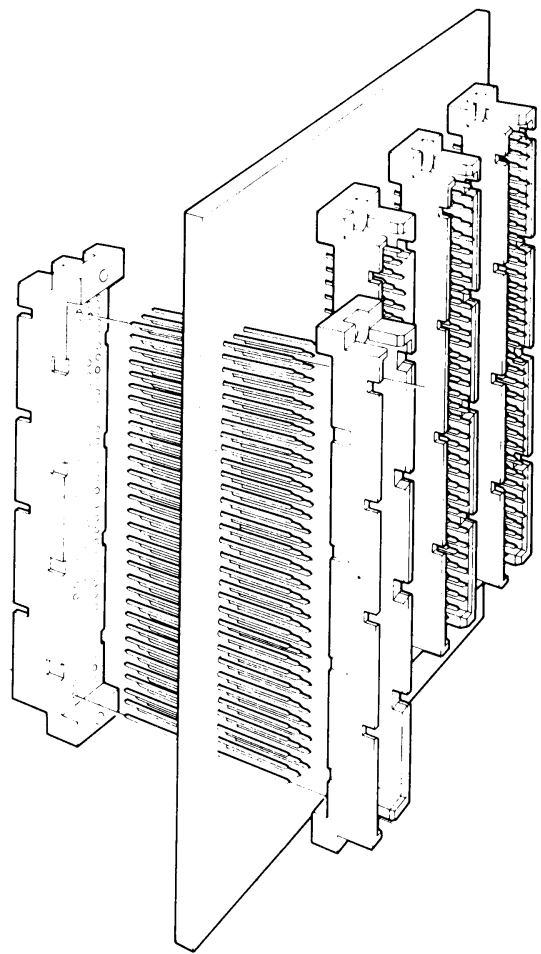

FIGURE 4 Arrangements of pins stacked in printed circuit board.

The second step will be to design and produce a preloaded press-fit male connector. Work is well under way and first products can be expected in the near future. With this approach, the technical problems can be coped with one at a time. Furthermore, a large part of the market can be supplied with a suitable connector system even though development work is still at an early stage.

The Berg Electronics press fit system today consists of:

(a) the "Bow-Tie" press-fit pin

(b) a variety of plastic shrouds and accessories

(c) a comprehensive range of application machines.

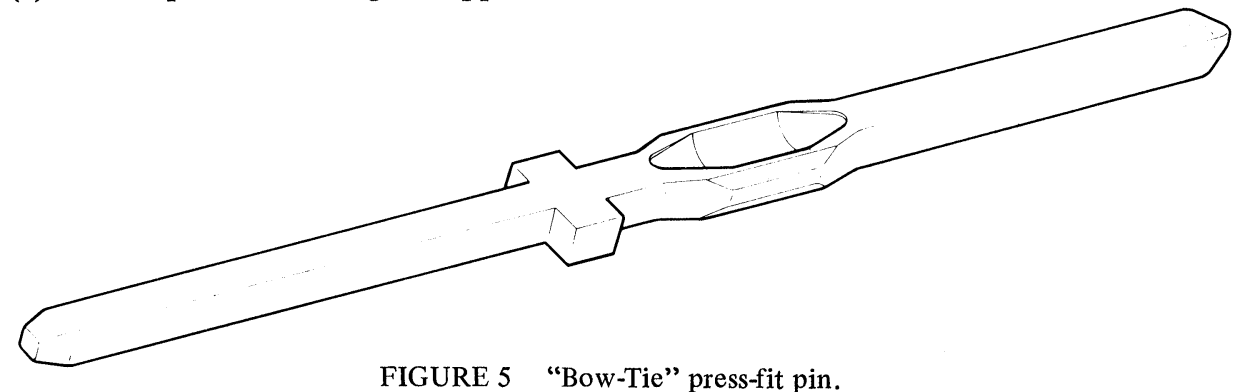

FIGURE 5 "Bow-Tie" press-fit pin.

The design of the "Bow-Tie" press fit pin, (Figure 5), is such that the compliance of the pin corresponds to holes in the printed circuit boards between 0,9 and $1,1 \mathrm{~mm}$. This makes it suitable for use with normal printed circuit boards. 
The actual compliance is found in the central web in the press-fit shape: when the pin is pressed into the hole, the outer sides of the shape move inward, whereby the web is allowed to shear in the middle. The two halves then slide over each other. The degree of shearing and deformation is of course dependant on the size of the hole. The compliant section's rounded corners and conically shaped entry section facilitate pin insertion and help to minimize hole deformation and damage to plating (see Figure 6).

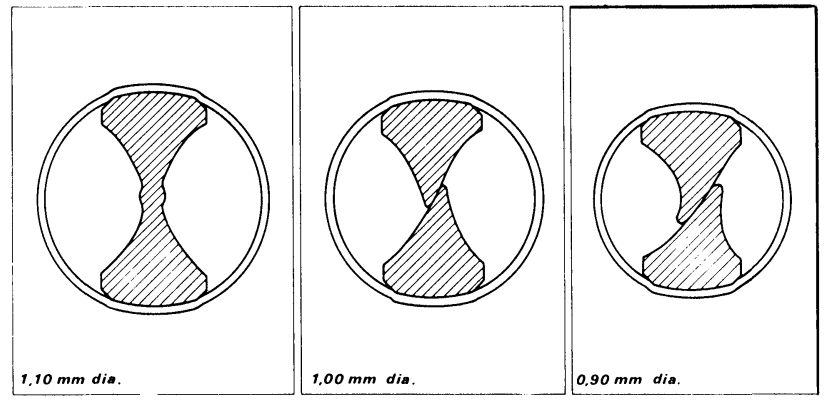

FIGURE 6 Effect of pressing pin into holes of different sizes.

\section{RESULTS}

In the following graphs the insertion and retention forces are depicted as a function of the hole diameter and for different parameters.

With a solid pin, insertion force increases rapidly with decrease in hole diameter (see Figure 7). This increase is a function of the increased contact zone. The graph was established for a printed circuit board thickness of $3,2 \mathrm{~mm}$ and shows clearly that solid pins can only be used in holes with a tolerance of $\pm 0,05 \mathrm{~mm}$.

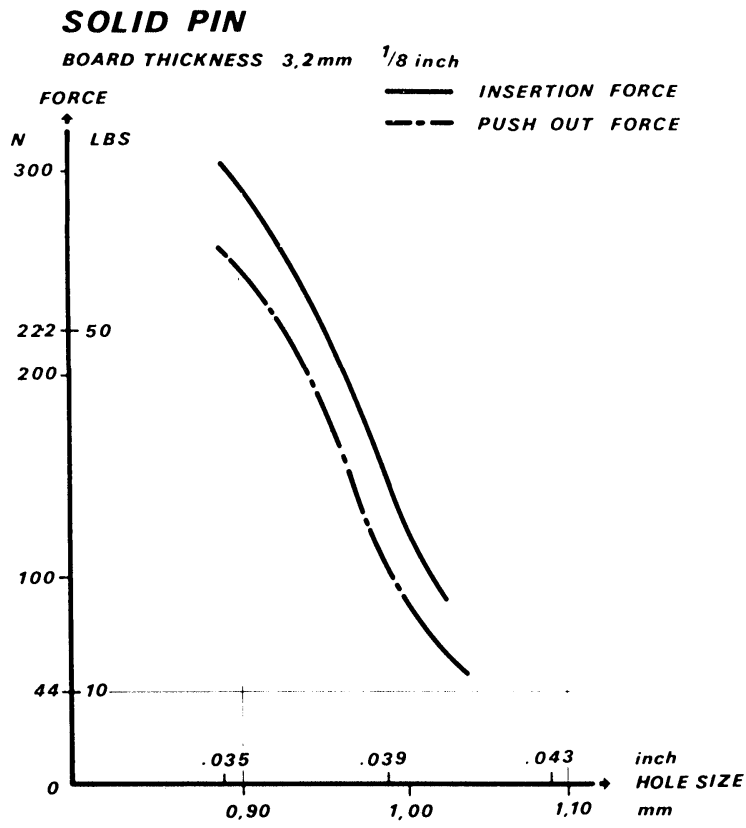

FIGURE 7 Insertion force vs hole size for solid pins. 
Under the same conditions, the Berg "Bow-Tie" pin has a much less pronounced curve slope. Insertion force is lower because of the conical entry section and the compliance of the pin itself. Retention force varies only slightly with hole diameter because of the pin's flexibility in large as well as small holes. The minimum retention force is largely superior to the industry requirement of $44 \mathrm{~N}$ (Figure 8).

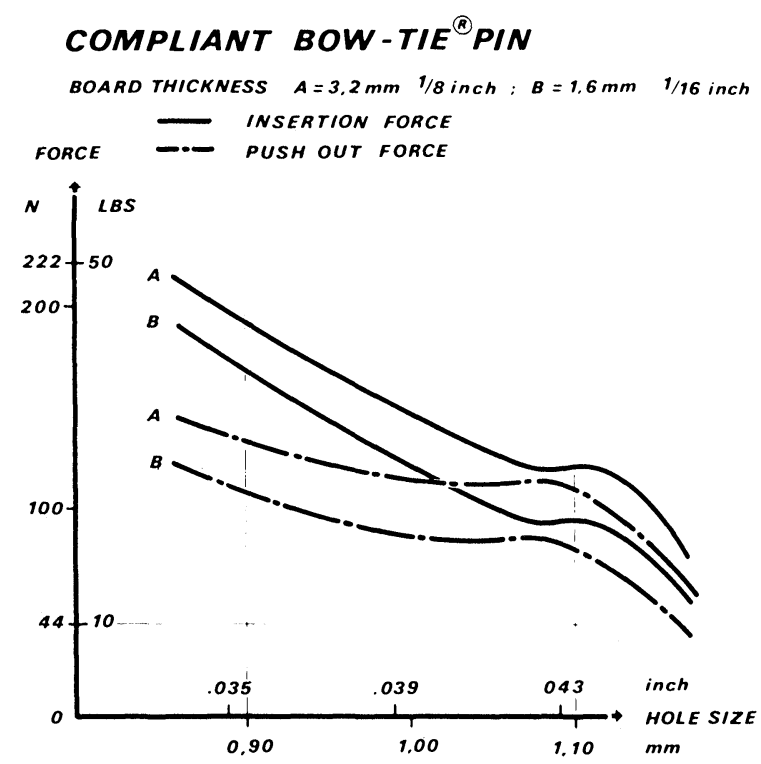

FIGURE 8 Insertion force vs hole size for "Bow-Tie" pins.

Another important criterion for a compliant press-fit pin is the retention force after repair. The "Bow-Tie" pin still fulfills the requirement after 5 insertions (Figure 9).

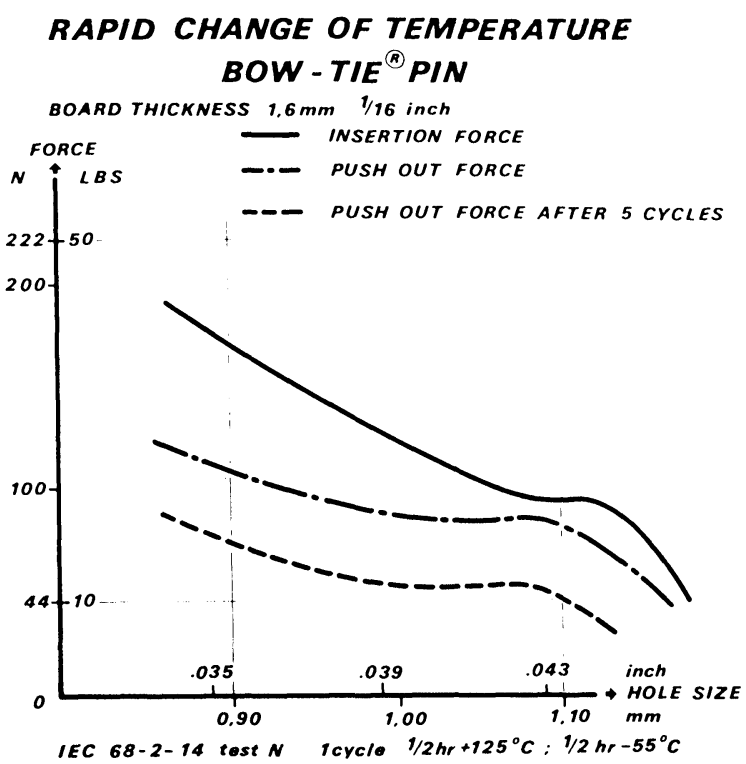

FIGURE 9 Insertion force vs hole size for "Bow-Tie" pins after 5 insertions. 
To determine the relaxation behaviour of the pin shape as a function of time, an accelerated ageing test was performed. After 5 rapid temperature change cycles, the retention force did indeed diminish but still remained well above the minimum values of the standard in the industry (see Figure 10). This is valid for board thicknesses of 3,2 and $1,6 \mathrm{~mm}$.

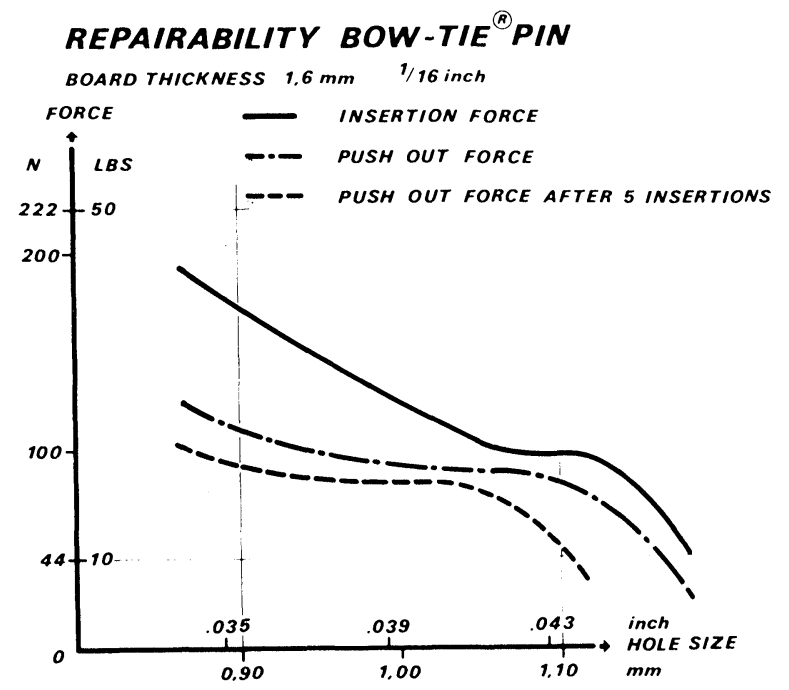

FIGURE 10 Insertion force vs hole size for "Bow-Tie" pins after 5 temperature cycles.

\section{APPLICATION EQUIPMENT}

In any press-fit system, the quality of the end result is influenced to a large extent by the application machine. Applied costs are closely linked to the production rates of the machine. Three different machines have been developed, plus a repair kit for individual pins. These are:

(a) A pneumatic mass-inserter with a production rate of 2500 pins an hour.

(b) A Pantograph machine that stakes up to 4000 terminals per hour.

(c) A computer-controlled, fully automatic machine, with rotating XY table, achieves a production rate of up to 25000 pins per hour.

The two bigger machines can stake press-fit pins in any pattern required. All three have many control functions as standard, while some special features can also be provided to comply with individual user requirements.

\section{CONCLUSIONS}

It is clear that press-fit techniques, with all the applied cost advantages they can bring to the user, are not a simple matter. Many variables of pin and printed circuit board together determine the practical and commercial viability of this new technology. At the same time, it should be emphasized that good solutions do exist in the market place. One such example is the Backpanel System discussed above in which innovative designs and techniques are used to solve connection problems in the most trouble free and cost effective way. 

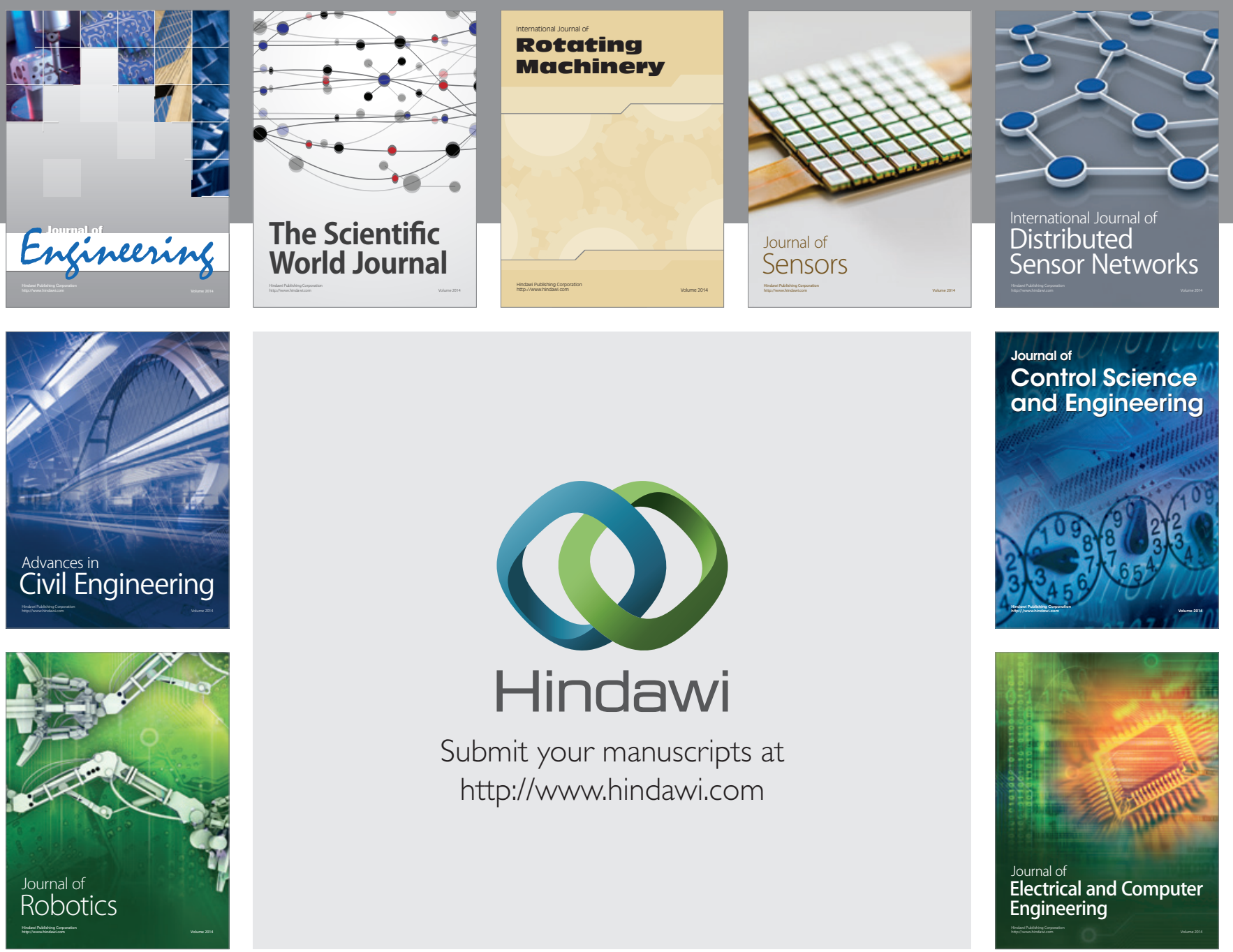

Submit your manuscripts at

http://www.hindawi.com
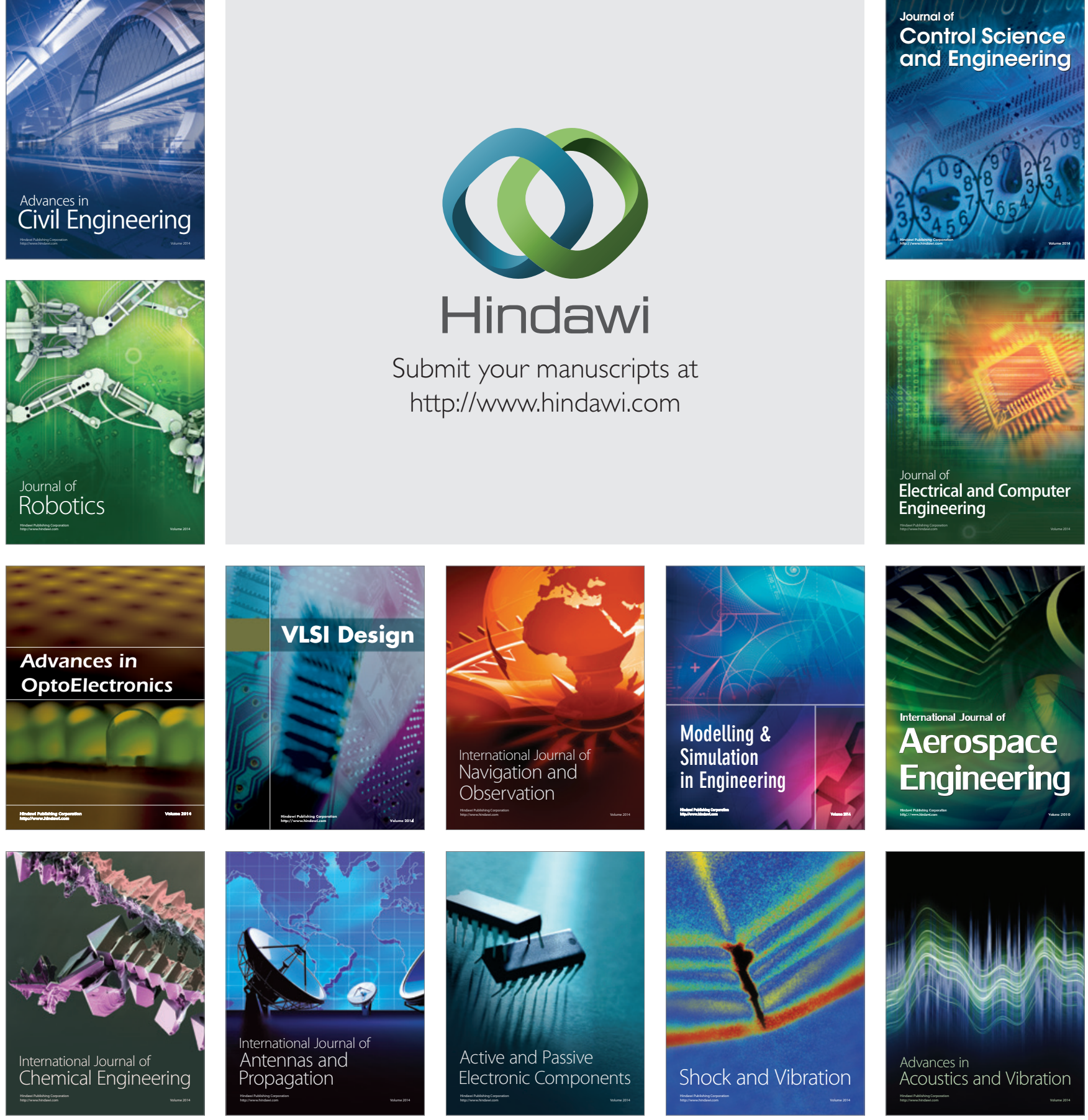